\title{
Phase transitions in the lattice model of intercalation
}

\author{
T.S.Mysakovych, V.O.Krasnov, I.V.Stasyuk \\ Institute for Condensed Matter Physics of the National Academy of Sciences of Ukraine, \\ 1 Svientsitskii Str., 79011 Lviv, Ukraine
}

Received November 10, 2008

\begin{abstract}
The lattice model which can be employed for the description of intercalation of ions in crystals is considered in this work. Pseudospin formalism is used in describing the interaction of electrons with ions. The possibility of hopping of intercalated ions between different positions is taken into account. The thermodynamics of the model is investigated in the mean field approximation. Phase diagrams are built. It is shown that at high values of the parameter of ion transfer, the phase transition to a modulated phase disappears.
\end{abstract}

Key words: intercalation, phase transition, pseudospin-electron model

PACS: $64.75 .+g, 71.20 . T x, 64.70 .-p$

Theoretical description of intercalation of ions in crystals appears to be an urgent problem because metal oxides are very promising electrode materials as hosts for ion (e. g., lithium) insertion. In [1-3] quantum chemical Hartree-Fock and density-functional calculations were performed to investigate lithium intercalation in $\mathrm{TiO}_{2}$ crystals. It was shown that $\mathrm{Li}$ is almost fully ionized once intercalated (Li looses its valence electron). It was revealed that reconstruction of electron spectrum at intercalation takes place. Thus, ion-electron interaction can play a significant role. At intercalation of lithium in $\mathrm{TiO}_{2}$, phase separation into Li-poor $\left(\mathrm{Li}_{\sim 0.01} \mathrm{TiO}_{2}\right)$ and Li-rich $\left(\mathrm{Li}_{\sim 0.5-0.6} \mathrm{TiO}_{2}\right)$ phases occurs. This two-phase behaviour leads to a constant value of electrochemical potential $[4,5]$. In [6], the theoretical investigation of intercalation was performed using the Hamiltonian which included the interaction between ions only. In our previous work [7] we formulated the pseudospinelectron model of intercalation and took into account the ion-electron interaction. It was shown that the effective attractive interaction between ions is formed due to the pseudospin-electron interaction and thus the condition of the appearance of phase transition was established. It was found that the capacity of the system increases near the phase transition point. In the present paper we consider the possibility of a transfer of intercalated ions and investigate the thermodynamics of the model.

The Hamiltonian of the model is written as follows:

$$
H=\sum_{i j} \Omega_{i j} S_{i}^{+} S_{j}^{-}+\sum_{i j \sigma} t_{i j} c_{i \sigma}^{+} c_{j \sigma}+\sum_{i \sigma}\left(g S_{i}^{z} n_{i \sigma}-\mu n_{i \sigma}\right)-\sum_{i} h S_{i}^{z} .
$$

The pseudospin variable $S_{i}^{z}$ takes two values; $S_{i}^{z}=1 / 2$ when there is a lithium ion in a site $i$ and $S_{i}^{z}=-1 / 2$ when there is no lithium ion, $c_{i \sigma}^{+}$and $c_{i \sigma}$ are electron creation and annihilation operators, respectively. We consider the possibility of ion and electron jumps between sites (the first and the second term in (1)) and interaction of electrons with lithium ions ( $g$ term); $\mu$ and $h$ play the role of chemical potentials of electrons and Li ions, respectively. The Hamiltonian is similar to the one used in describing a system of coexisting itinerant electrons and local pairs when the creation and destruction operators for local pairs (hard-core bosons) obey the Pauli spin 1/2 commutation rules (for example, see [9]). However, it should be noted that in [9] the chemical potential of the local pairs and itinerant electrons was the same and the regime of a fixed total number of particles was used.

To investigate the thermodynamics of the model we use the following simplification:

$$
\begin{aligned}
g n_{i} S_{i}^{z} & \rightarrow g\left\langle n_{i}\right\rangle S_{i}^{z}+g n_{i}\left\langle S_{i}^{z}\right\rangle-g\left\langle n_{i}\right\rangle\left\langle S_{i}^{z}\right\rangle, \\
\Omega S_{i}^{+} S_{j}^{-} & \rightarrow \Omega\left\langle S_{i}^{+}\right\rangle S_{j}^{-}+\Omega S_{i}^{+}\left\langle S_{j}^{-}\right\rangle-\Omega\left\langle S_{i}^{+}\right\rangle\left\langle S_{j}^{-}\right\rangle,
\end{aligned}
$$


where the pseudospin-electron interaction and ion transfer are taken into account in the spirit of the mean field approximation (MFA) through internal self-consistent fields which act on electrons and pseudospins.

We consider the possibility of the formation of a modulated (chess-board - like) phase and that is why we introduce two sublattices: $\left\langle\sum_{\sigma} n_{i \alpha \sigma}\right\rangle=n_{\alpha},\left\langle S_{i \alpha}^{z}\right\rangle=\eta_{\alpha}$, where $\alpha=1,2$ is a sublattice index, $i$ is an elementary cell index. The Hamiltonian of the model in the MFA is as follows:

$$
\begin{aligned}
H^{M F A}= & \sum_{i \alpha \sigma}\left(g \eta_{\alpha}-\mu\right) n_{i \alpha \sigma}+\sum_{i \alpha}\left(g n_{\alpha}-h\right) S_{i \alpha}^{z}+\sum_{i \alpha, j \beta} t_{i j}^{\alpha \beta} c_{i \alpha \sigma}^{+} c_{j \beta \sigma}+\sum_{\alpha \beta i} 2 \Omega^{\alpha \beta}\left\langle S_{\alpha}^{x}\right\rangle S_{i \beta}^{x} \\
& -g \sum_{i \alpha} n_{\alpha} \eta_{\alpha}-N \Omega\left\langle S_{1}^{x}\right\rangle\left\langle S_{2}^{x}\right\rangle
\end{aligned}
$$

where $N$ is the number of the lattice sites, $\Omega \equiv \Omega^{12}=\Omega^{21}=\sum_{i} \Omega_{i j}^{12} ; \Omega^{11}=\Omega^{22}=t^{11}=t^{22}=0$.

To diagonalize the Hamiltonian, we pass to $\boldsymbol{k}$-representation and perform the transformations

$$
\begin{aligned}
& c_{\boldsymbol{k} 1 \sigma}=\cos \phi \tilde{c}_{\boldsymbol{k} 1 \sigma}+\sin \phi \tilde{c}_{\boldsymbol{k} 2 \sigma}, \\
& c_{\boldsymbol{k} 2 \sigma}=-\sin \phi \tilde{c}_{\boldsymbol{k} 1 \sigma}+\cos \phi \tilde{c}_{\boldsymbol{k} 2 \sigma}, \\
& \sin 2 \phi=\frac{t_{\boldsymbol{k}}}{\sqrt{\left(g \frac{\eta_{1}-\eta_{2}}{2}\right)^{2}+t_{\boldsymbol{k}}^{2}}}, \cos 2 \phi=\frac{-g \frac{\eta_{1}-\eta_{2}}{2}}{\sqrt{\left(g \frac{\eta_{1}-\eta_{2}}{2}\right)^{2}+t_{\boldsymbol{k}}^{2}}} ; \\
& S_{i \alpha}^{z}=\sigma_{i \alpha}^{z} \cos \theta_{\alpha}+\sigma_{i \alpha}^{x} \sin \theta_{\alpha}, \quad S_{i \alpha}^{x}=\sigma_{i \alpha}^{x} \cos \theta_{\alpha}-\sigma_{i \alpha}^{z} \sin \theta_{\alpha}, \\
& \sin \theta_{\alpha}=\frac{2 \Omega\left\langle S_{\beta}^{x}\right\rangle}{\tilde{\lambda}_{\alpha}}, \quad \cos \theta_{\alpha}=\frac{h-g n_{\alpha}}{\tilde{\lambda}_{\alpha}}, \\
& \tilde{\lambda}_{\alpha}=\sqrt{\left(g n_{\alpha}-h\right)^{2}+\left(2 \Omega\left\langle S_{\beta}^{x}\right\rangle\right)^{2}}, \quad \alpha \neq \beta .
\end{aligned}
$$

Then the Hamiltonian is as follows:

$$
\begin{aligned}
H & =\sum_{\alpha \sigma \boldsymbol{k}}\left(\lambda_{\boldsymbol{k} \alpha}-\mu\right) \tilde{n}_{\boldsymbol{k} \alpha \sigma}-\sum_{i \alpha} \tilde{\lambda}_{\alpha} \sigma_{i \alpha}^{z}-g \frac{N}{2}\left(n_{1} \eta_{1}+n_{2} \eta_{2}\right)-N \Omega\left\langle S_{1}^{x}\right\rangle\left\langle S_{2}^{x}\right\rangle, \\
\lambda_{\boldsymbol{k} \alpha} & =g \frac{\eta_{1}+\eta_{2}}{2}+(-1)^{\alpha} \sqrt{\left(g \frac{\eta_{1}-\eta_{2}}{2}\right)^{2}+t_{\boldsymbol{k}}^{2}} .
\end{aligned}
$$

The electron band changes its position at intercalation (see [7] for more details). Besides, the doubling of a unit cell leads to the splitting in the electron spectrum [8].

The equations for electron and ion concentrations can be obtained using the diagonal Hamiltonian (6):

$$
\begin{aligned}
& n_{\alpha}=\frac{1}{N} \sum_{\boldsymbol{k} \sigma}\left(\frac{1+\cos 2 \phi}{2}\left(\mathrm{e}^{\frac{\lambda_{\boldsymbol{k} \alpha}-\mu}{T}}+1\right)^{-1}+\frac{1-\cos 2 \phi}{2}\left(\mathrm{e}^{\frac{\lambda_{\boldsymbol{k} \beta}-\mu}{T}}+1\right)^{-1}\right) \\
& \eta_{\alpha}=\frac{h-g n_{\alpha}}{2 \tilde{\lambda}_{\alpha}} \tanh \left(\frac{\beta \tilde{\lambda}_{\alpha}}{2}\right) \\
& \left\langle S_{\alpha}^{x}\right\rangle=-\frac{2 \Omega\left\langle S_{\beta}^{x}\right\rangle}{2 \tilde{\lambda}_{\alpha}} \tanh \left(\frac{\beta \tilde{\lambda}_{\alpha}}{2}\right)
\end{aligned}
$$

The grand canonical potential can be written as follows:

$$
\begin{aligned}
\frac{\Phi}{\frac{N}{2}=} & -\frac{T}{N} \sum_{\boldsymbol{k}, \sigma} \ln \left(\left(1+\mathrm{e}^{\frac{\mu-\lambda_{\boldsymbol{k} 1}}{T}}\right)\left(1+\mathrm{e}^{\frac{\mu-\lambda_{\boldsymbol{k} 2}}{T}}\right)\right) \\
& -T \ln \left(4 \cosh \frac{\beta \tilde{\lambda}_{1}}{2} \cosh \frac{\beta \tilde{\lambda}_{2}}{2}\right)-g\left(n_{1} \eta_{1}+n_{2} \eta_{2}\right)-2 \Omega\left\langle S_{1}^{x}\right\rangle\left\langle S_{2}^{x}\right\rangle .
\end{aligned}
$$


The obtained set of equations was solved numerically (we use the grand canonical potential (9) to find thermodynamically stable states).

In calculations we used the semielliptic density of states, $\rho(\epsilon)=\frac{2}{\pi W^{2}} \sqrt{W^{2}-\epsilon^{2}},-W<\epsilon<W$, where $W$ is a half-width of the electron band (in our calculations we put $W=1$ ).

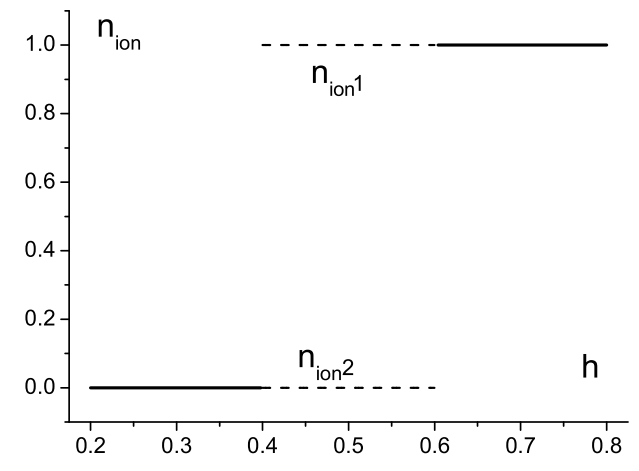

(a)

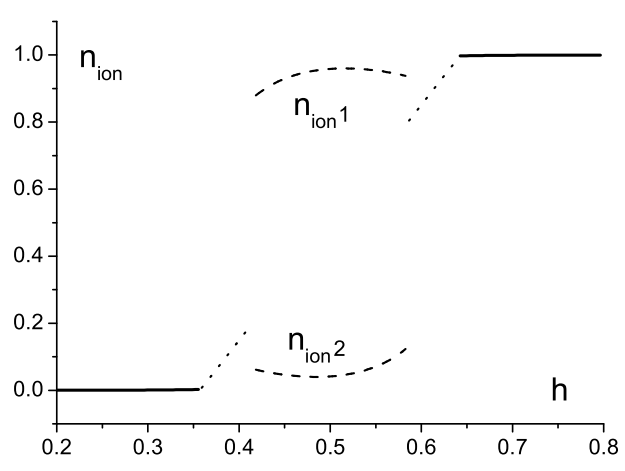

(b)

Figure 1. The dependence of ion concentration on chemical potential of the ions. The parameter values are: $g=0.5, W=1, \mu=0, T=0.05, \Omega=0(a), \Omega=0.3(b)$.

In figure 1 the dependence of the ion $n_{\operatorname{ion} \alpha}=\eta_{\alpha}+1 / 2$ concentration on the chemical potential of ions is shown (a similar behaviour is obtained for the electron concentration) in the regime of a fixed chemical potential of electrons for the following set of parameters: $W=1, g=0.5, T=$ $0.05, \mu=0, \Omega=0(a), \Omega=0.3(b)$. Solid line corresponds to the case of a uniform phase with $\left\langle S^{x}\right\rangle=0$, dotted line relates to the case of a uniform phase with $\left\langle S^{x}\right\rangle \neq 0$, dashed line relates to the case of the modulated phase $\left(\left\langle S_{1,2}^{x}\right\rangle=0\right.$ for the case $\Omega=0$ and $\left\langle S_{1,2}^{x}\right\rangle \neq 0$ for the case $\Omega=0.3$ ). From figure 1 we can see that the system undergoes a phase transition from a uniform to a modulated phase $\left(n_{\text {ion } 1}\right.$ and $n_{\text {ion } 2}$ are the ion concentrations in two sublattices in a modulated phase). The mean value $\left\langle S^{x}\right\rangle$ is equal to zero in the case $\Omega=0$ and at $\Omega \neq 0$ an additional phase with $\left\langle S^{x}\right\rangle \neq 0$ can appear. When the system goes from a uniform phase to a modulated phase, the jumps of ion and electron concentrations occur. This means that in the regime of fixed concentrations, the phase separation in two phases (uniform and modulated) with different ion and electron concentrations takes place [10].

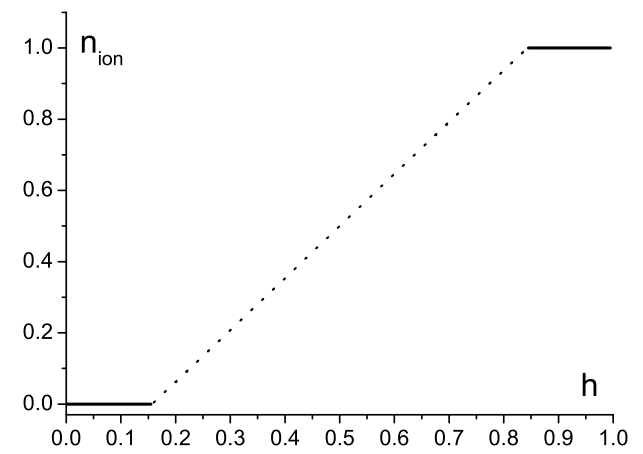

(a)

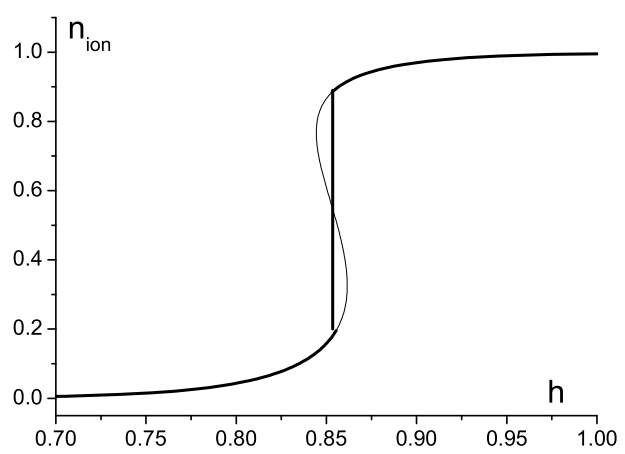

(b)

Figure 2. The dependence of ion concentration on chemical potential of the ions. The parameter values are: $g=0.5, W=1, T=0.05, \Omega=0.5, \mu=0(a), \Omega=0, \mu=0.6(b)$. 
In figure 2 the dependence of ion concentration on chemical potential of ions is shown for the cases $\mu=0, \Omega=0.5$ (a) and $\mu=0.6, \Omega=0(\mathrm{~b})$. We can see that at high values of the parameter of ion transfer $\Omega$, the phase transition to a modulated phase (and the phase separation in the regime of the fixed concentrations) disappears. When the chemical potential is placed near the band edges, the phase transition of the first order between two uniform phases with jumps of the ion and electron concentration can appear; this is illustrated in figure 2(b).

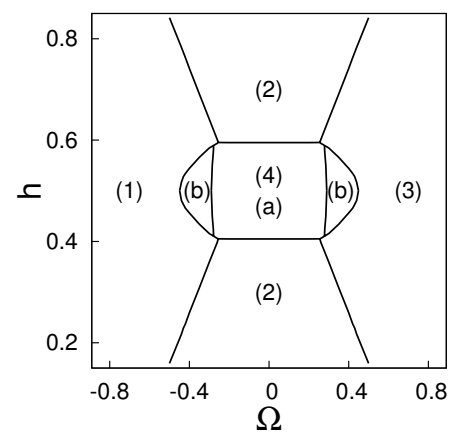

(a)

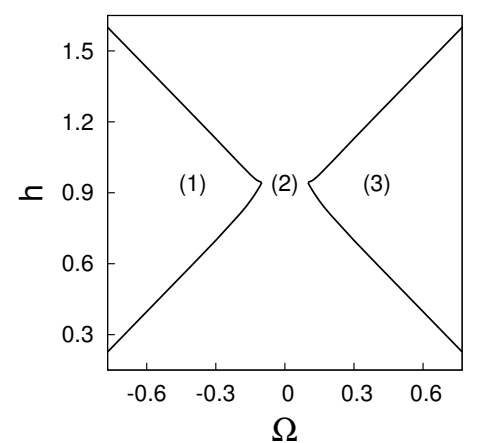

(b)

Figure 3. Phase diagrams in the $(h-\Omega)$ plane $((\mathrm{a})-\mu=0,(\mathrm{~b})-\mu=0.8)$. The parameter values are: $g=0.5, W=1, T=0.05$. (1),(3) - uniform phase with $\left\langle S^{x}\right\rangle \neq 0\left((1):\left\langle S_{1}^{x}\right\rangle=\left\langle S_{2}^{x}\right\rangle\right.$, (3): $\left.\left\langle S_{1}^{x}\right\rangle=-\left\langle S_{2}^{x}\right\rangle\right)$; (2) - uniform phase with $\left\langle S^{x}\right\rangle=0$; (4) - modulated phase ((a): $\left\langle S_{1,2}^{x}\right\rangle=0$, (b): $\left.\left\langle S_{1,2}^{x}\right\rangle \neq 0\right)$.

In figure 3 the $h-\Omega$ phase diagrams are shown for the cases $\mu=0$ (at the centre of the band) and $\mu=0.8 W$ (near the band edge). In figure 4 the $h-\mu$ phase diagram is shown. It is easy to see from figures 3,4 that at high values of the parameter of the ion transfer $\Omega$, the only possible phases are the uniform phases with $\left\langle S^{x}\right\rangle \neq 0$ and $\left\langle S^{x}\right\rangle=0$.

Thus, we may conclude that the ion transfer leads to the disappearance of phase transitions with jumps of the ion and electron concentrations. Besides, the new phase with $\left\langle S^{x}\right\rangle \neq 0$ appears due to the presence of ion hopping between sites; this phase is an analogy to a superfluid phase in the systems of hard-core bosons. To investigate this phase in detail we should examine the behaviour of the conductivity and other characteristics of the system. This is the task for future investigations.

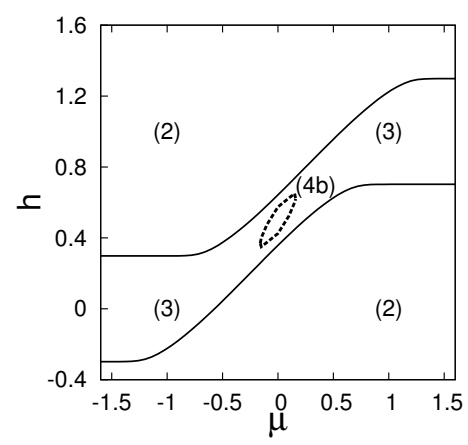

Figure 4. Phase diagram in the $(h-\mu)$ plane. The parameter values are: $g=0.5, W=1$, $T=0.05, \Omega=0.3$. The phase notations are the same as in figure 3 . 


\section{References}

1. Stashans A., Lunell S., Bergström R., Hagfeldt A., Lindquist S.-E., Phys. Rev. B, 1996, 53, 159.

2. Koudriachova M.V., Harrison N.M., de Leeuw W., Phys. Rev. Lett, 2001, 86, 1275.

3. Koudriachova M.V., Harrison N.M., de Leeuw W., Phys. Rev. B, 2002, 65, 235423.

4. Wagemaker M., van de Krol R., Kentgens A.P.M., van Well A.A., Mulder F.M., J. Am. Chem. Soc., 2001, 123, 11454.

5. Wagemaker M., Kearley G.J., van Well A.A., Mutka H., Mulder F.M., J. Am. Chem. Soc., 2003, 125, 840.

6. Jung K.-N., Pyun S.-I., Kim S.-W., J. Power Sources, 2003, 119-121, 637.

7. Mysakovych T.S., Stasyuk I.V. J. Phys. Studies, 2007, 11, 195.

8. Stasyuk I.V., Mysakovych T.S. J. Phys. Studies, 2001, 5, 268.

9. Robaszkiewicz S., Pawlowski G. J. Superconductivity, 2004, 17, 37.

10. Stasyuk I.V., Mysakovych T.S. Condens. Matter Phys., 2001, 5, 268.

\section{Фазові переходи в гратковій моделі інтеркаляції}

\section{Т.С.Мисакович, В.О.Краснов, І.В.Стасюк}

Інститут фізики конденсованих систем НАН України, 79011 Львів, вул. Свєнціцького, 1

Отримано 10 листопада 2008 р.

В даній роботі розглядається граткова модель, яка може бути застосована для опису інтеркаляції іонів в кристали. Використано псевдоспіновий формалізм для опису взаємодії електронів з іонами та враховано можливість перескоків іонів між різними позиціями. Досліджено термодинаміку моделі в наближенні середнього поля. Побудовано фазові діаграми. Показано, що при великих значеннях іонного переносу фазовий перехід у модульовану фазу зникає.

Ключові слова: інтеркаляція, фазові переходи, псевдоспін-електронна модель

PACS: $64.75 .+g, 71.20 . T x, 64.70 .-p$ 
